\title{
COVID-19 in patients with juvenile idiopathic arthritis: frequency and severity
}

\author{
Oksana Boyarchuk ${ }^{1}$ ID , Lilya Predyk ${ }^{1}$, Iryna Yuryk ${ }^{2}$ \\ 1Department of Children's Diseases and Pediatric Surgery, I. Horbachevsky Ternopil National Medical University, Ternopil, Ukraine \\ ${ }^{2}$ Regional Children's Hospital, Ternopil, Ukraine
}

\begin{abstract}
The aim of the study was to determine the frequency and the course of COVID-19 infection in children with juvenile idiopathic arthritis (JIA). The study involved 51 patients with JIA aged 2 to 18 years. Evidence of COVID-19 was found in 10 (19.6\%) patients with JIA. COVID-19 infection occurred more often in patients with systemic arthritis (OR $=6.1667,95 \% \mathrm{Cl}: 1.2053-31.5511, p=0.0289)$. The course of COVID-19 infection in patients with JIA was generally similar to the course in the pediatric population, despite immunosuppressive therapy. In 3 out of 10 patients the infection caused an exacerbation of JIA, which required therapy escalation.
\end{abstract}

Key words: COVID-19 infection, juvenile idiopathic arthritis, immunosuppressive therapy.

\section{Introduction}

Most children with COVID-19 have mild symptoms and favorable outcomes, which has been shown in some studies [1, 2], with the exception of multisystem inflammatory syndrome in children (MIS-C) associated with SARS-CoV-2 and hyperinflammation in COVID-19 with life-threatening manifestations and long-term sequelae $[3,4]$.

On the other hand, the COVID-19 pandemic has raised a number of questions for rheumatologists, including pediatric rheumatologists, which relate primarily to a higher risk of COVID-19 and more severe course of this infection in patients with rheumatic diseases considering common immunosuppressive therapy that have a negative impact on the immune system $[5,6]$.

Research data are contradictory as some of them point to greater susceptibility to COVID-19 (almost 3 times) in patients with rheumatic musculoskeletal diseases [3], while others showed that the risk of severe COVID-19 was associated with older age, male gender, obesity, hypertension, and chronic kidney disease $[7,8]$ that was already identified in the general population, as well as current or prior treatment with glucocorticosteroids and use of some other antirheumatic drugs (rituximab and mycophenolate mofetil) $[5,8,9]$.

Juvenile idiopathic arthritis (JIA) is the most frequent chronic rheumatic disease in children. It remains unclear whether children with JIA are at a higher risk and whether we need to change the treatment of JIA in patients with COVID-19 infection. Publications about the COVID-19 course in patients with JIA are limited, and there are only a few recommendations for pediatric rheumatologists [10-12].

The aim of the study was to determine the frequency of COVID-19 infection in children with JIA and the course of COVID-19 on the background of JIA while immunosuppressive therapy was used.

\section{Material and methods}

Children with JIA who were being followed up in Ternopil Regional Children's Hospital were interviewed between October 2020 and January 2021. The study involved 51 patients with JIA aged 2 to 18 years: 18 of them were observed during inpatient or outpatient visits to the hospital, while the rest were contacted through 
Table I. Characteristics of patients with juvenile idiopathic arthritis

\begin{tabular}{|lccc|}
\hline Characteristic & JIA + COVID-19, $n=10$ & JIA without COVID-19, $n=41$ & JIA (all patients), $n=51$ \\
\cline { 2 - 4 } & $n(\%)$ & $n(\%)$ & $n(\%)$ \\
\hline Age, years, mean (range) & $10.8(6-15)$ & $12.6(2-18)$ & $12.3(2-18)$ \\
\hline Female & $5(50)$ & $21(51.2)$ & $26(51)$ \\
\hline Systemic JIA & $4(40)$ & $4(9.8)$ & $8(15.7)$ \\
\hline JIA, oligoarthritis & $2(20)$ & $24(58.5)$ & $26(51)$ \\
\hline JIA, polyarthritis & $2(20)$ & $7(17.1)$ & $9(17.6)$ \\
\hline JIA, enthesitis related & $2(20)$ & $5(12.2)$ & $7(13.7)$ \\
\hline Psoriatic JIA & - & $1(2.4)$ & $1(2)$ \\
\hline Treatment & $8(80)$ & $36(87.8)$ & $43(84.3)$ \\
\hline Methotrexate & $4(40)[0 / 4]$ & $7(17.1)[2 / 5]$ & $11(21.6)[2 / 9]$ \\
\hline Steroids [current/prior] & $3(30)$ & $3(7.3)$ & $6(11.8)$ \\
\hline Adalimumab & 0 & $6(14.6)$ & $6(11.8)$ \\
\hline Tocilizumab & & & \\
\hline
\end{tabular}

a telephone-based survey. Data about COVID-19 exposure and/or diagnosis were collected.

Evidence of COVID-19 infection was typical clinical presentation and exposure to a confirmed COVID-19 family case and/or positive for SARS-CoV-2 infection by PCR and/or positive by serology. Patients with evidence of COVID-19 were interviewed about the course of the disease. Specifically, all the symptoms, duration, treatment, and complications were recorded. Baseline patients' characteristics were obtained from medical records.

\section{Results}

Evidence of COVID-19 was found in 10 (19.6\%) patients with JIA. Two patients were positive for SARS CoV-2 infection by PCR, 6 patients were positive by serology and 2 patients had a close exposure to a confirmed by PCR COVID-19 family case and clinical symptoms of COVID-19.

Systemic arthritis was recorded in 4 cases, while oligoarthritis, polyarthritis and enthesitis related arthritis were recorded in 2 children (Table I).

Five patients have received methotrexate, and 3 children have received methotrexate and adalimumab. Four patients with systemic IIA previously received systemic corticosteroids. COVID-19 infection prevailed in patients with systemic arthritis; all of them previously received corticosteroids. The course of COVID-19 in 8 (80\%) children was mild and did not require specific treatment.

Fever was reported in 8 children; in two of them it was $39-40^{\circ} \mathrm{C}$ on the first day of infection, in others up to $38-38.5^{\circ} \mathrm{C}$. Fever lasted for $3-5$ days. Cough was observed in 4 children, anosmia in 3 , taste loss in 2 , arthral- gia in 5, and weakness in 2 children. Only one patient presented with pneumonia and received antibiotic therapy. This patient was in remission of systemic arthritis and was being followed without treatment. Neither of the patients required hospitalization for COVID-19.

However, in 3 cases JIA flare was reported on the background of infection and continued after the symptoms of COVID-19, which required prescription of glucocorticoids in 2 cases and non-steroidal anti-inflammatory drugs were prescribed in 1 case. In 1 case there was worsening of seizures after COVID-19 infection which was attributed to the neurological comorbidity [13].

Family contact history of COVID-19 was reported in 11 cases of JIA; in 7 of them there was other evidence of COVID-19 in patients and in 4 cases it was absent. Children who were in close contact with family members with COVID-19, but without evidence of infection, also received immunosuppressants: methotrexate (3 cases), methotrexate with adalimumab (1 case).

The results demonstrated that COVID-19 occurred more often in patients with systemic arthritis $(\mathrm{OR}=6.1667$, $95 \% \mathrm{Cl}: 1.2053-31.5511, p=0.0289)$. We did not observe a severe course of COVID-19 in patients with JIA, including those on immunosuppressive therapy, counting immunobiological drugs, as was also reported in previous studies $[11,14]$.

Our data demonstrated that patients with systemic arthritis, even having previously taken corticosteroid therapy, are at a higher risk for SARS-CoV-2 infection, possibly due to the peculiarities of the immune response to the virus in this category of patients, which requires further study. 
Current or prior glucocorticoid use was found to be a risk factor for COVID-19 hospitalization in adult patients with inflammatory rheumatic disease in other studies $[8,9]$.

Research recently conducted in Turkey reported that among 345 patients with JIA there were 8 patients with confirmed COVID-19 and supported low frequency of severe disease complications [11], which is consistent with the results of our study.

The limitation of this study is the small group of COVID-10 positive children, which indicates that a confirmatory study in a larger cohort of patients is required. The patients with JIA and COVID-19 should be followed up for a longer time to detect possible distant exacerbations after infection.

\section{Conclusions}

The course of COVID-19 infection in patients with JIA is generally similar to the course of COVID-19 in the pediatric population, despite receiving immunosuppressive therapy. However, in 3 out of 10 patients the infection caused an exacerbation of JIA, which required therapy escalation.

The author declares no conflict of interest.

\section{References}

1. Patel NA. Pediatric COVID-19: systematic review of the literature. Am J Otolaryngol 2020; 41: 102573, DOI: 10.1016/j.amjoto. 2020.102573.

2. Toptan T, Ciesek S, Hoehl S. Pediatrics and COVID-19. Adv Exp Med Biol 2021; 1318: 197-208, DOI: 10.1007/978-3-030-63761-3_12.

3. Henderson LA, Canna SW, Friedman KG, et al. American College of Rheumatology clinical guidance for multisystem inflammatory syndrome in children associated with SARSCoV-2 and hyperinflammation in pediatric COVID-19: version 2. Arthritis Rheumatol 2021; 73: e13-e29, DOI: 10.1002/art. 41616.

4. Sancho-Shimizu V, Brodin P, Cobat A, et al. SARS-CoV-2-related MIS-C: a key to the viral and genetic causes of Kawasaki disease? J Exp Med 2021; 218: e20210446, DOI: 10.1084/jem. 20210446.

5. Makowska J, Styrzyński F. Between COVID-19 severity and its prevention - what should rheumatologists be aware of? Reumatologia 2021; 59: 1-2, DOI: 10.5114/reum.2021.103941.

6. Leszczyński P. COVID-19: a short message to rheumatologists. Reumatologia 2020; 58: 130-133, DOI: 10.5114/reum. 2020.96685.

7. Zhong J, Shen G, Yang H, et al. COVID-19 in patients with rheumatic disease in Hubei province, China: a multicentre retrospective observational study. Lancet Rheumatol 2020; 2: e557-e564, DOI: 10.1016/S2665-9913(20)30227-7.

8. FAI2R/SFR/SNFMI/SOFREMIP/CRI/IMIDIATE consortium and contributors. Severity of COVID-19 and survival in patients with rheumatic and inflammatory diseases: data from the French RMD COVID-19 cohort of 694 patients. Ann Rheum Dis 2020; 80: 527-538, DOI: 10.1136/annrheumdis-2020-218310 [Online ahead of print].

9. Hasseli R, Mueller-Ladner U, Hoyer BF, et al. Older age, comorbidity, glucocorticoid use and disease activity are risk factors for COVID-19 hospitalisation in patients with inflammatory rheumatic and musculoskeletal diseases. RMD Open 2021; 7: e001464, DOI: 10.1136/rmdopen-2020-001464.

10. Licciardi F, Giani T, Baldini L, et al. COVID-19 and what pediatric rheumatologists should know: a review from a highly affected country. Pediatr Rheumatol Online J 2020; 18: 35, DOI: 10.1186/ s12969-020-00422-z.

11. Yildiz M, Haslak F, Adrovic A, et al. The frequency and clinical course of COVID-19 infection in children with juvenile idiopathic arthritis. Clin Exp Rheumatol 2020; 38: 1271-1272.

12. Calvo C, Udaondo C; Rheumatic Diseases EPICO-AEP Working Group. COVID-19 in children with rheumatic diseases in the Spanish National Cohort EPICO-AEP. J Rheumatol 2021, DOI: 10.3899/jrheum.201548 [Online ahead of print].

13. Boyarchuk O, Kovalchuk T, Kovalchuk N, Chubata O. Clinical variability of the systemic juvenile idiopathic arthritis course: literature review based on case series. Reumatologia 2020; 58: 436-443, DOI: 10.5114/reum.2020.10201.

14. Filocamo G, Minoia F, Carbogno S, et al. Absence of severe complications from SARS-CoV-2 infection in children with rheumatic diseases treated with biologic drugs. J Rheumatol 2020, DOI: 10.3899/jrheum.200483 [Online ahead of print]. 